\title{
POLARIZATION OF QUASARS
}

\author{
R. G. CONWAY \\ Nuffield Radio Astronomy Laboratories, Jodrell Bank, Cheshire, Great Britain
}

I report here work done at Jodrell Bank by $\mathrm{Mr}$ John Gilbert and myself at a wavelength of $49 \mathrm{~cm}$, on the degree of linear polarization, $m_{49}$. For most sources the polarization is considerably reduced from its short wavelength value. We presume that most of this depolarization is by Faraday rotation, or rather by Faraday dispersion, between different parts of the source.

The Australian workers have found little if any correlation of short wavelength polarization with other parameters. However, at a wavelength of $49 \mathrm{~cm}$ there is good correlation with spectral index, $\alpha$ : flat-spectrum or 'young' quasars show systematically higher polarization than steep spectrum or 'old' quasars.

Because this effect does not show at short wavelengths, it is a difference of depolarization rate. The Faraday dispersion of steep quasars must be mostly $>8 \mathrm{rad} \mathrm{m}^{-2}$, whereas the Faraday dispersion of flat quasars is mostly below this figure, either because of a deficit of thermal electrons, or for some other reason.

The reduction of $m$ with increasing wavelength suggests that there ought to be a correlation of $m$ with redshift $z$, because as $z$ increases the emitted wavelength decreases, and hence $m$ should increase. For $z=2$ the emitted wavelength is $16 \mathrm{~cm}$. We have predicted curves of $m$ vs $z$ both for flat and for steep-spectrum quasars, assuming that all quasars out to $z=2.5$ have the properties of the local ones for which $z<1$.

For each category, the polarization at high $z$ is less than expected by a factor of about 3. Because the effect is present for both categories of quasar it can hardly be an evolutionary effect. It is more likely that distant quasars are covered by some sort of Faraday screen. For example a spiral galaxy would give a rotation measure of about 60 for a line of sight going through a spiral arm, but a much smaller value for a line of sight passing through an interarm gap. Actually our observations require a Faraday dispersion in excess of $80 \mathrm{rad} \mathrm{m}^{-2}$. It is possible that field galaxies could provide this, but rather more likely that the Faraday screen is associated with the quasar.

In either case, one might expect the screen to show absorption lines. Out of 25 quasars with $z>1.25$ we found seven with absorption lines. Those with absorption lines have $\bar{m}_{49}=0.56 \pm 0.21 \%$, while those without average $1.38 \pm 0.29 \%$.

Hence it appears that the same clouds cause Faraday polarization and absorption lines. The product $N_{e} B L$ for such a cloud must be about $10^{-4} \mathrm{~cm}^{-3} \mathrm{G} \mathrm{pc}$.

I also wish to announce that our most recent measurements made in March of this year, have shown circular polarization in 5 out of 21 quasars, with one further possible case.

We used the interferometer at Jodrell Bank of Mark I and Mark II telescopes, integrating for $8 \mathrm{hr}$ per source. Measurements on 3C 295 were used as calibration. 
The apparent value of circular polarization of 3C 147 and 3C 123 agreed precisely with $3 \mathrm{C} 295$ to $0.03 \%$, and we believe that the instrumental correction is right to this accuracy, and that all three sources are unpolarized.

Two sources have polarization which is clearly above noise, at $3.0 \sigma$ and $3.4 \sigma$ respectively. Four others have values from $1 \sigma$ to $2 \sigma$ which on $\theta$ could not credit on statistics alone, but they do all have the common characteristic of possessing a flat spectrum.

According to simple theory by Legg and Westfold the corresponding value of $B$ is $10^{-3} \mathrm{G}$ for $0.2 \%$ polarization. If the field is irregular then $|B|>10^{-3} \mathrm{G}$.

\section{Discussion}

Komesaroff: If the circular polarization is proportional to $f^{-1 / 2}$ this does not prove the correctness of the $10^{-3} \mathrm{G}$ deduced from the Legg and Westfold paper. Gleeson et al. have shown that for a dipole field which would in general yield a much lower circular polarization than the uniform field discussed by the previous authors, the circular polarization may still show an $f^{-1 / 2}$ dependence. The deduced $10^{-3} \mathrm{G}$ is most probably a lower limit.

Kellermann: At least for CTA 102 the assumption of a power law energy distribution for the relativistic electrons is correct as shown by the short wavelength radio emission where the source is optically thin or has a power law spectrum.

Also for this source the value of the magnetic field derived from the circular polarization measurements is close to that estimated from the measured peak brightness temperature and self-absorption cutoff frequency.

Terzian: It is interesting to see that CTA 102 shows circular polarization. Several years ago CTA 102 was reported to be a periodic variable radio source; however, several series of observations performed with linearly polarized feeds showed no variability. The observations showing the variability were performed with circularly polarized feeds. In your observations did you notice any variability?

Conway: Our integration time was about $8 \mathrm{hr}$, during which time we noticed no change.

Scheuer: Is there any correlation between the rotation measure and the occurrence of absorption lines in QSOs?

Conway: I shall have to reply privately to this, which I agree is a very interesting question. May I comment that the difficulty in correlating anything against the presence of absorption lines is that the number of reported absorption lines is increasing very rapidly with time just now. 